\title{
Development of a Prototype Electronic Alternator for DIN/PACS Environment and its Evaluation
}

\author{
H. S. Choi ${ }^{1}$, H. W. Park ${ }^{1}$, D. R. Haynor ${ }^{2}$, and Y. Kim ${ }^{1}$ \\ ${ }^{1}$ Department of Electrical Engineering \\ ${ }^{2}$ Department of Radiology \\ University of Washington \\ Seattle, WA 98195
}

\begin{abstract}
A prototype electronic alternator (EA) has been implemented utilizing an advanced image processing workstation and evaluated for its clinical acceptability as a primary diagnostic workstation. The workstation is based on a PIXAR II image computer and is a node on the University of Washington Digital Imaging Network and Picture Archiving and Communications System (DIN/PACS, hereafter PACS). The goals of this study are to utilize the unique features of the workstation (large image memory, a high-speed parallel transfer disk (PTD), and a $2560 \mathrm{x} 2048$ high-resolution image monitor) to demonstrate the feasibility of a limited model of the EA as a primary diagnostic workstation in the PACS environment, to evaluate its capability in image viewing and diagnosis, and to assess the feasibility of an icon-based user interface to select and rearrange images on the monitor. In this paper, various characteristics of the prototype EA such as image display and processing performance, image storage capacity, and functional specification as well as the results of a clinical evaluation are presented. The main emphasis of the clinical evaluation was on system speed, image quality, and user preference for an icon-based user interface.
\end{abstract}

\section{INTRODUCTION}

In a radiology department with PACS ${ }^{1,2}$, the image viewing and processing workstation (the workstation, hereafter) becomes an electronic substitute for the conventional multipanel film alternator ${ }^{3}$ or simple light box, and the performance of the workstation is a critical factor for both productivity and diagnostic quality. Ideally, the workstation should be able to compete with the conventional film alternator in both data processing speed and display quality. However, this is not realistic, at least at present, primarily for technical reasons. The speed with which a film alternator can handle images exceeds that of most existing workstations. The time required for an alternator to present a new panel of eight $14^{\prime \prime} \times 17^{\prime \prime}$ films, which corresponds to approximately 60 Mbytes of data (assuming each image is represented by a $1760 \times 2140$ computed radiography-type spatial resolution with each pixel taking two bytes), is between 1 and 10 seconds. A PACS workstation offers advantages over the film alternator in other aspects, such as flexible access to patients' images and ancillary information and the capability of interactively enhancing images utilizing window and level $(\mathrm{W} / \mathrm{L})$ and other image processing techniques.

Among a variety of workstation design issues ${ }^{4,5,6}$, the most important factors are system response time (speed), image quality, and user interface. The speed with which the workstation executes certain functions, such as image retrieval and display and image database management, plays a large role in its clinical acceptability because of its effect on efficiency and productivity. The advantages of a good user interface go beyond efficiency. In many hospital environments, personnel are constantly changing. As a result, there is a continual need for training of new users. A well-designed user interface allows easy learning of the system, reducing training time and errors. Finally, image quality has direct effect on diagnostic accuracy and so is crucial for both radiologists and referring physicians.

This work is supported in part by the U.S. Army Medical Research Acquisition Activity of the U.S. Army Medical Research and Development Command, Contract No. DAMD17-86-C-6145. The views, opinions, and/or findings contained in this document are those of the authors and should not be construed as official Department of Army policy or decision unless so designated by other documentation. 
This paper presents a prototype electronic alternator (EA) developed utilizing a commercially available imaging workstation. Also, this paper summarizes its clinical evaluation results which provide performance criteria for the future design of the PACS workstations, particularly for the three design issues mentioned above.

The EA has been developed as a single-screen, high-performance workstation which would be usable for primary analysis of cases by radiologists and/or referring physicians. The EA hardware features a PIXAR II image computer with a commercially available high-speed parallel transfer disk (PTD) (Maximum Strategy, San Jose, CA). Currently, the EA is a node of the University of Washington PACS ${ }^{1}$, and is interfaced to the AT\&T CommView PACS system via standard Ethernet using the TCP/IP network protocol. The EA user interface is a Macintosh-like mouse-driven window-based system and has been developed utilizing the SUN window development system. Image manipulation is facilitated by image icons and a pictorial directory, a set of minified image icons which represent actual images in the image database. The EA allows the user to manipulate two sets of images for side-by-side comparison, and offers flexible image layout.

In order to evaluate the EA for clinical acceptability as a primary diagnostic workstation, we designed a laboratory evaluation session and used a questionnaire in order to collect the evaluation results. Seven radiologists from the University of Washington participated in the laboratory sessions and answered the questionnaire with a variety of comments. In section 2, we describe the design and implementation of the EA and the user interface, followed by the evaluation methodology in section 3. In section 4 , we present the clinical evaluation results and discuss the results and some design issues for the future PACS workstations.

\section{ELECTRONIC ALTERNATOR}

\subsection{Hardware}

Figure 1 shows the hardware configuration of the EA. The heart of the EA is a PIXAR II image computer with a SUN 3/180 as the host computer. Images are displayed on a $2560 \times 2048 \times 8$-bit Megascan monochrome monitor and a $1280 \times 1024$ Sony full-color monitor. A SUN's $1152 \times 900 \times 1$-bit console is used for the user interface. The PIXAR II has a total of 48 Mpixels of image memory with 12-bit pixel depth. Each image monitor is driven by a separate display processor. Although the depth of the image memory is 12 bits, the PIXAR II uses only eight bits for display on the Megascan monitor. The most significant bit is used as a sign bit, and the following eight bits are used for display with the low-order three bits truncated. Thus, the images must be scaled either before or after loading into image memory. For fast retrieval of processed and unprocessed images from the local image database, a parallel transfer disk (PTD) with a data transfer rate of up to $8 \mathrm{Mbytes} / \mathrm{sec}$ is connected directly to the PIXAR II through a high-speed local bus. In the PTD, four high-speed disks work simultaneously during data retrieval. The major interface to the PACS system is implemented utilizing the SUN's Ethernet port, which is connected to the AT\&T CommView system via a gateway. The communication protocol is standard TCP/IP.

\subsection{Functional Specifications}

The functional level specifications of the EA include: 1) a user interface which facilitates efficient use of the EA hardware and software features, 2) image manipulation and processing functions, and 3) a network interface to the central image database.

The EA user interface was developed using the SUN window development system which is a development toolkit for the window-based user interface. The user interface consists of two major functional modules, the image management module and clinical image viewing and processing module. The image management module performs local image database management as well as image downloading from the central image database. Currently, only downloading of computed radiography (CR) images from the central database to the EA is supported. The clinical image viewing and processing module displays images on both $2 \mathrm{~K}$ and $1 \mathrm{~K}$ image monitors, calls image processing functions, analyzes displayed images, and stores processed images in the local image database which is implemented with the PTD.

Consistency was emphasized in the design of the user interface. The SUN mouse has three buttons on it. The 




Figure 1. EA hardware configuration 
left button is dedicated to the initiation of various functions, while the right button is used to stop the selected functions. The middle button was used for switching between $2 \times 2$ and $1 \times 1$ display modes, which are described later. The keyboard usage for the interface was minimized. In the clinical image viewing and processing mode, the keyboard need not be used at all. Figure 2 shows the function menu layout of the user interface in this mode.

The Megascan monitor can be used in either $2 \times 2$ or $1 \times 1$ display mode. In the $2 \times 2$ display mode, which is mainly used for comparison studies, it can display four $1024 \times 1024$ low-resolution CR images. The low-resolution images are precomputed and stored in the local database (PTD) during the image downloading from the central image database into the EA. In order to avoid visual aliasing in the course of the spatial resolution reduction, which can be caused by sharp edges in the images, the EA applies a lowpass filter to full-resolution images before down-sampling. In the $1 \times 1$ display mode, the Megascan displays one full-resolution image. The switch between the $1 \times 1$ and $2 \times$ 2 display modes is done by a single mouse click. Since a low-resolution image and the corresponding full-resolution image are always loaded in the PIXAR II's invisible image memory simultaneously, the switching between the $1 \mathrm{x}$ 1 and $2 \times 2$ display modes is instantaneous. The EA can load up to four low and four full-resolution CR images in the PIXAR II's image memory.

Image selection and rearrangement utilize image icons. An image icon is a miniature image ( $128 \times 128 \times 1$ bit $)$ which represents a full-resolution image in the local image database. To obtain an image icon, a full-resolution image is lowpass-filtered and decimated followed by binary thresholding. The decimation resolution is chosen dynamically based on the image size of the full-resolution image since the size of CR images varies. This process is performed during the image downloading from the central image database and is transparent to the user.

The EA can handle two sets of images of a patient; each set is called an examination and can contain up to four images. Once either one or two examinations have been selected by the user, the EA displays the image icons of all available images in the selected examinations in the pictorial directory. Up to eight image icons from two examinations can be displayed in the pictorial directory. Image layout in the $2 \times 2$ display mode is easily configured by utilizing the pictorial directory and the monitor icon. The monitor icon represents the Megascan display in the $2 \times 2$ display mode and shows the corresponding image icons of the images which are actually displayed or will be displayed on the Megascan monitor. The pictorial directory and monitor icon are shown in Fig. 2. A set of eight image icons at the bottom of the image icon area in the figure is the pictorial directory and the monitor icon is on top of the pictorial directory. In addition, the figure shows mouse selectable buttons for various functions, a list of patients available in the local image database, a list of examinations of a selected patient, and a list of images.

The display layout is controlled by selecting an image icon in the pictorial directory and then selecting a destination location in the monitor icon using the mouse. When a destination is selected, the selected image icon in the pictorial directory is copied to the selected location in the monitor icon. Images can be displayed in any location. Actual image loading into the PIXAR II's image memory is not initiated until the user clicks the image load button. This feature may save image loading time if the user wants to reconfigure image layout on the monitor.

The EA supports both global and local W/L. The global W/L changes the window and level of the whole screen while the local $\mathrm{W} / \mathrm{L}$ changes the window and level of only a single image in the $2 \times 2$ display mode. The global $\mathrm{W} / \mathrm{L}$ works only in the $1 \times 1$ display mode. Once the W/L button is selected, horizontal and vertical mouse movement adjusts the window and level, respectively. Global W/L was implemented utilizing the hardware display lookup table of the PIXAR II's video board. Since the Megascan only displays the upper 8-bit information of 11-bit stored images, the global W/L parameters lie between 0 and 255 .

The ability to adjust the window and level of individual image in the $2 \times 2$ display mode is very important for comparison studies. W/L implementation was more complicated with the $2 \times 2$ display mode since there is no hardware feature that supports region of interest (ROI) based video lookup table operations in the PIXAR II. We implemented the local $\mathrm{W} / \mathrm{L}$ by software emulation. Since the software $\mathrm{W} / \mathrm{L}$ is destructive (when the window and level of an image is changed, actual gray levels are modified), the EA loads another set of low-resolution images in the invisible image memory during initial image loading, from which the window and level adjusted images can be calculated. When the local W/L function is first initiated, the default target image is the one in the upper-left 


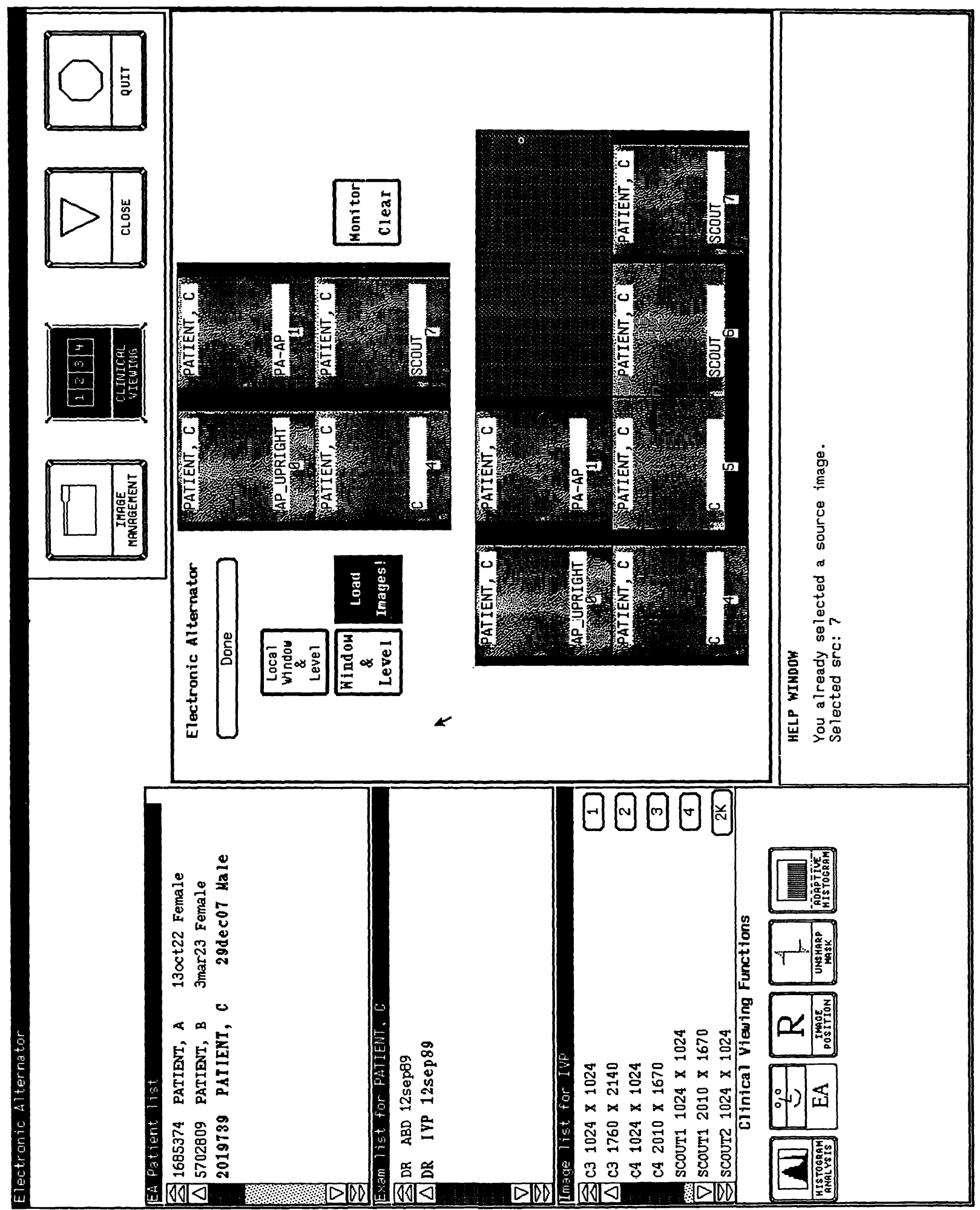

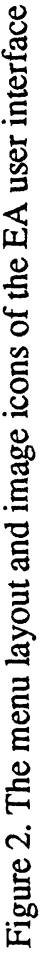


quadrant. The target image is easily changed using a pop-up menu which can be brought up by a mouse click.

When the W/L parameters of an image are adjusted, the updated values are stored in a data structure assigned to each image, so that the EA can restore the previous window and level when the image is reselected for display. This feature applies to both global and local W/L. The EA supports adaptive histogram equalization and unsharp masking algorithms as image processing tools. The adaptive histogram equalization was implemented utilizing PIXAR's image processing library. It can be applied to any size of images up to $2560 \times 2048$ and can undersample images with variable rate for fast estimation of the histograms.

\section{EVALUATION}

The evaluation of the EA emphasized three aspects: 1) the increased speed of image access possible with the PTD and large image memory, 2) the improved spatial resolution possible with the $2560 \times 2048$ high-resolution monitor, and 3) the clinical acceptability of an icon-based approach to selection and rearrangement of images on the image monitor. The EA was evaluated by seven radiologists from the University of Washington Medical Center. Two of these were chest radiologists, one was a bone radiologist, and four performed general radiology. All had a certain amount of experience in using computers, primarily for word processing, and all had used a Macintosh.

For clinical evaluation, we used three patient cases, all of which were CR studies performed at the University of Washington Hospital using a Philips Computed Radiography (PCR) system. These cases were selected based on the following criteria: 1) the number of examinations was more than one with a certain time interval between the examinations and 2) the number of images in each examination was less than four (to meet current hardware constraints). Those three cases were a chest, an abdomen, and an extremity case. All patients had two examinations. The number of images for each patient ranged from 4 to 6 . Before the evaluation sessions, the three cases were downloaded from the CommView's central database, so that the evaluators did not have to wait for images to be transmitted from the PACS central database. During the downloading, the EA automatically generated the image icons and low-resolution images.

Drapes were used to exclude extraneous light and to minimize the monitor glare. A person familiar with the EA was present for each evaluation session and was immediately available to assist with any interface problems. After a brief training session (using the chest case), the evaluators were asked to read the other two cases. While the evaluators were reading the images, the session assistant guided the evaluators so that they used all the system features which would be asked about in the post-session evaluation questionnaire. After the reading session, the evaluators filled out a questionnaire. Each question asked for a numerical score for the clinical acceptability of the designated aspect of the EA. While the evaluators were filling out the questionnaire, they were allowed to go back to any images they wanted to view again.

\section{EVALUATION RESULTS AND DISCUSSIONS}

\subsection{Image Manipulation Performance}

The system performance was measured with $1760 \times 2140 \mathrm{CR}$ images. Initial loading of a four-image examination from the local image database (PTD) took $10.17 \mathrm{sec}$. Such an examination includes eight low-resolution images (four for display and four for W/L backup) and four full-resolution images. Downloading an image from the central database to the local database took approximately 5 minutes. This includes the time for preparing a low-resolution image and an image icon. Switching image display mode from the $2 \times 2$ mode to the $1 \times 1$ mode took $0.66 \mathrm{sec}$, while going to the $2 \times 2$ mode from the $1 \times 1$ mode took $3.86 \mathrm{sec}$. The difference in times was mainly due to the software emulation of the local $\mathrm{W} / \mathrm{L}$ which was applied to all four low-resolution images to restore the previous window and level. The $\mathrm{W} / \mathrm{L}$ computation time for one low-resolution image was about $0.66 \mathrm{sec}$. The time to load a full-resolution image from the PTD into the image memory was $1.75 \mathrm{sec}$ including the system overhead. Convolution with a $3 \times 3$ kernel took $1.94 \mathrm{sec}$. and the unsharp masking with a $11 \times 11$ kernel took 12.9 sec. Currently, the unsharp masking supports only low-resolution images. 


\subsection{Clinical Evaluation Results}

All ratings in the evaluation questionnaire were expressed on a 6-position scale ranging from -3 (totally unacceptable for clinical work) to +3 (totally acceptable). A description of the questionnaire and the scores associated with each question are given in the Appendix.

The speed of the system, in bringing full-resolution $(2 \mathrm{~K} \times 2 \mathrm{~K})$ images to the monitor, was rated as +3 by five radiologists and +2 by the other two; thus, it was fully acceptable. The speed in bringing a panel of four $1 \mathrm{~K} \times 1 \mathrm{~K}$ images was also rated comparable to the speed of full-resolution images. Both the low-resolution images and the high-resolution images were rated as moderately acceptable for diagnosis (mean $=1.50$ and 1.57 , respectively). One radiologist commented that the availability of a zoom feature could have made the image quality at high-resolution more acceptable. The awkwardness of the mouse-driven W/L may also have adversely affected the ratings. Some of the evaluators complained mildly about the display speed of low-resolution $(2 \times 2)$ images. This was due to software emulation of local W/L control, as described above.

The ability to compare multiple studies was rated as moderately acceptable (mean $=2.14$ ). Some radiologists commented that, for image rearrangement on the image monitor, dragging an image icon from the pictorial directory to the monitor icon may be more intuitive than clicking the mouse with the cursor on a source image icon and then clicking again on a destination location in the monitor icon. The image icons, which had only 1-bit depth resolution, were felt to be rather poor representations of the corresponding images, with three of the radiologists assigning a quality of $-1($ mean $=0.42)$.

Adjustment of window and level was done by moving the mouse to the left or right (window adjustment) or away from/toward the user (for level adjustment). The effects of the lack of ROI-based video lookup table hardware were felt particularly strongly with the low-resolution images. The software emulation of the local window and level setting in the $2 \times 2$ display mode apparently was not well accepted. The time required to update the window and level of a low-resolution image was approximately $0.66 \mathrm{sec}$., which was felt to be unacceptable (mean $=0.28)$. When a single image was displayed at full resolution, it was possible to adjust the window and level interactively since this was implemented utilizing a hardware lookup table, and this speed was judged to be quite acceptable $($ mean $=2.35)$.

Spatial resolution of the images was felt to be acceptable (mean $=2.43$ ). The contrast resolution was judged to be somewhat less acceptable (mean $=1.07$ ), primarily because of the compromises that had to be made to utilize the 8-bit display hardware. Image quality was judged to be slightly inferior to film (mean $=1.83$ ), but it was rated comparable to CR hard copy $($ mean $=2.50)$.

Most of the evaluators' comments on the icon-based user interface were very positive. Many of them had a certain amount of familiarity with a Macintosh-type user interface, so that it was easy for them to get used to the mousebased user interface of the EA. Currently, the EA does not load images into the EA image memory until the user specifically requests that the image be loaded. Several users wanted some sort of intelligent default image display when a patient is selected at the beginning of a reading session.

The recommended monitor layout of the Megascan is portrait mode (2048 x 2560), rather than landscape mode (2560 x 2048), since most of the CR images are generated in portrait mode. One of the most frequently used CR image formats is $1760 \times 2140$. When an image in this format is displayed in full-resolution, 92 rows at the bottom of the image are truncated in the landscape mode display.

\section{CONCLUSIONS}

In this paper, we presented a prototype electronic alternator and its clinical evaluation. The evaluation results show that using currently available hardware, access to locally available images can be fast enough to be acceptable to radiologists and that an icon-based interface is quite acceptable, and indeed preferred, by radiologists.

Several hardware-based limitations became apparent, however. An 8-bit display processor is not adequate for use as 
an electronic alternator for biomedical images. Another serious limitation was the lack of tools for the development of window-based user interfaces which use the high-resolution image monitor (Megascan). As a result, the current implementation utilizes a separate SUN monitor for the text and image icon display. With the separate text and image monitors, the user could not concentrate on the image monitor all the time. In order to improve the user's productivity, merging the text and image monitor is crucial, especially for a user interface which utilizes a mouse as an interactive input device.

\section{APPENDIX}

This appendix describes the questionnaire which each EA evaluator was asked to fill out after his evaluation session. Each question asked for the clinical acceptability of a particular EA feature using the following scale:

$\begin{aligned} 3 & \text { Totally acceptable } \\ 2 & \text { Moderately acceptable } \\ 1 & \text { Barely acceptable } \\ -1 & \text { Slightly unacceptable } \\ -2 & \text { Moderately unacceptable } \\ -3 & \text { Totally unacceptable }\end{aligned}$

The definition of clinical acceptability on this scale was left to the individual evaluator. The mean score and standard deviation (in parenthesis) associated with each question are given below.

\section{SPEED}

How satisfactory was the speed with which low-resolution images appeared? $2.57(0.49)$

How satisfactory was the speed with which full-resolution images appeared? $2.71(0.45)$

How adequate were the low-resolution images for image comparison? $\quad 1.50$ (1.16)

How adequate were the full-resolution images for primary diagnosis? 1.57 (1.59)

How clinically acceptable was the time required to rearrange the layout of images on the monitor? $2.14(0.79)$

How satisfactory was the multi-study feature? $2.14(0.63)$

\section{ICONIC IMAGE DIRECTORY}

How useful was the iconic image directory? $2.00(0.53)$

How adequate was the quality of the image icons? $\quad 0.42(1.59)$

\section{WINDOW AND LEVEL}

\section{General}

How intuitive was the method of adjusting window and level? $\quad 1.50(1.10)$

How appropriate was the input device? $2.07(0.41)$

Window and Level Adjustment - single 2K image

How appropriate was the response time for window and level adjustment on the entire screen? $\quad 2.35(0.69)$

Window and Level Adjustment - single 1K image (out of four)

How appropriate was the response time for window and level adjustment for a single image? $\quad 0.28$ (1.84)

\section{IMAGE QUALITY}

How acceptable was the spatial resolution of the image at full resolution? $2.43(0.49)$

How acceptable was the contrast (fineness of gray scale) at full resolution? $1.07(2.30)$

How acceptable was the amount of noise in the full-resolution images? 

Abdominal image?
$1.71(1.58)$
Extremity image?
$2.00(1.31)$

How acceptable was the amount of noise in the low-resolution images?
Abdominal image?
$2.43(0.49)$
Extremity image?
$2.57(0.49)$

How does the image quality compare to conventional film? $1.83(0.58)$

How does image quality compare to CR hard copy ? $2.50(0.43)$

\section{REFERENCES}

1. R. K. Panwar, C. S. Wang, L. A. DeSoto, H. W. Park, and Y. Kim, "UW PACS Prototype Performance Measurements, Computer Model, and Simulation," SPIE Medical Imaging IV, Vol. 1234, in press, 1990.

2. R. E. Braudes, S. K. Mun, J. Sibert, J. Schnizlein, and S. Horii, "Workstation Modelling and Development: Clinical Definition of a Picture Archiving and Communications System (PACS) User Interface," SPIE Medical Imaging III, Vol. 1093, pp. 376-386, 1989.

3. K. G. O'Malley and J. A. Giunta, "The Alternator: Determination of its Fundamental Features, as a Basis for Design of a PACS," SPIE Medical Imaging II, Vol. 914, pp. 988-994, 1988.

4. J. C. Gee, L. A. DeSoto, Y. Kim, D. R. Haynor, and J. W. Loop, "User Interface Design for a Radiological Imaging Workstation," SPIE Medical Imaging III, Vol. 1093, pp. 122-132, 1989.

5. D. Beard, S. Pizer, D. Rogers, R. Cromartie, S. Desirazu, S. Ramanathan, and R. Rubin, "A Prototype Singlescreen PACS Console Development Using Human Computer Interaction Techniques," SPIE Medical Imaging, Vol. 767, pp. 646-653, 1987.

6. Y. Kim, J. B. Fahy, L. A. DeSoto, D. R. Haynor, and J. W. Loop, "Development of a PC-based Radiological Imaging Workstation," SPIE Medical Imaging II, Vol. 914, pp. 1257-1264, 1988. 